Review began 09/09/2021 Review ended 02/01/2022 Published 02/03/2022

๑) Copyright 2022

Saven et al. This is an open access article distributed under the terms of the Creative Commons Attribution License CC-BY 4.0. which permits unrestricted use, distribution, and reproduction in any medium, provided the original author and source are credited.

\section{Co-prescription of Dual-Antiplatelet Therapy and Proton Pump Inhibitors: Current Guidelines}

\author{
Hannah Saven ${ }^{1}$, Lynna Zhong ${ }^{1}$, Isabel M. McFarlane ${ }^{2}$ \\ 1. Internal Medicine, State University of New York (SUNY) Downstate College of Medicine, New York, USA 2. Internal \\ Medicine, State University of New York (SUNY) Downstate Medical Center, New York, USA
}

Corresponding author: Isabel M. McFarlane, isabel.mcfarlane@downstate.edu

\begin{abstract}
Dual-antiplatelet therapy (DAPT) prevents thrombotic complications associated with coronary artery disease, acute coronary syndrome, and stent thrombosis following the percutaneous coronary intervention or coronary artery bypass grafting. When initiating DAPT, the risk of thrombosis must be balanced with the increased risk of upper gastrointestinal bleed (UGIB). Proton-pump inhibitors (PPIs) are concurrently prescribed with DAPT to reduce bleeding risk.
\end{abstract}

In this review, we discuss the benefits and potential complications of DAPT/PPI co-prescription. The only large international randomized control trial (RCT), Clopidogrel and the Optimization of Gastrointestinal Events Trial (COGENT), shows robust evidence that PPIs are a safe and effective method to reduce the risk of bleeding in patients on DAPT. However, more large-scale RCTs are needed to study potential long-term effects and draw a stronger conclusion on this topic.

Categories: Cardiology, Internal Medicine, Gastroenterology

Keywords: dapt/ppi prescription, cyp450 pathway, upper gastrointestinal bleed (ugib), proton-pump inhibitors (ppi), dual-antiplatelet therapy (dapt)

\section{Introduction And Background}

Dual-antiplatelet therapy (DAPT) is the indicated long-term therapy for preventing thrombotic complications associated with coronary artery disease (CAD) and acute coronary syndrome (ACS), and prevention of stent thrombosis following percutaneous coronary intervention (PCI) or coronary artery bypass grafting (CABG) following ST-segment elevation myocardial infarction (STEMI) or non-ST elevation myocardial infarction (NSTEMI) with OR intervention for six months [1,2]. DAPT typically consists of lowdose aspirin in combination with a P2Y12 inhibitor, such as clopidogrel, ticagrelor, or prasugrel, to produce an anti-thrombotic effect. The use of DAPT in treating ACS has led to improved outcomes for patients, especially with the development of more potent P2Y12 inhibitors [3]. Therefore, the increased use of drugeluting stents for patients with CAD has led to a significant increase in DAPT utilization to prevent stent thrombosis [4]. Stent thrombosis can result in a significant increase in death $[4,5]$. The administration of DAPT has decreased the risk of stent thrombosis in patients who underwent stent placement [6]. In addition, DAPT has been associated with a reduced risk of myocardial infarction (MI) and stroke [6].

Clinicians have become wary of balancing the bleeding risks associated with DAPT with the risk of stent thrombosis plus other major adverse cardiovascular and cerebrovascular events (MACCE) [7].

Gastrointestinal bleeding, particularly upper gastrointestinal bleed (UGIB), is one of the most common serious bleeding adverse effects of antiplatelet therapy, which has led to the concurrent prescription of proton pump inhibitors (PPIs). However, the use of PPI as gastrointestinal (GI) prophylaxis in the context of DAPT is controversial. This conflicting conclusion and the lack of more randomized control clinical trials (RCTs) have made the safety and efficacy of PPI for GI bleeding prophylaxis for patients on DAPT questionable. In this review, we will discuss the mechanism of DAPT, bleeding risks and epidemiology, and the evidence regarding PPI prophylaxis with concurrent DAPT prescription. This review will consider current guidelines from various medical societies, detail pharmacological mechanisms, and interactions of these medications, and consider studies from the last five years that have built upon the initial data concerning DAPT and PPI co-prescription.

\section{Review}

\section{Methods}

We used PubMed to review the literature published between 2016 and 2021 about concurrent DAPT and PPI co-prescription. We chose to review articles from the last five years to limit this review to the most recent literature and keep discussion applicable to current guidelines and practice. The review still included Clopidogrel and the Optimization of Gastrointestinal Events Trial (COGENT), which was published in 2010, because this study is integral to this discussion about DAPT and PPI co-prescription. Searched phrases include "gastrointestinal bleed and antiplatelet therapy," "gastroprotection and antiplatelet therapy," "PPI 
use and antiplatelet therapy,” “DAPT PPI,” and “GI bleeding and CVD.” Duplicate articles were disregarded. Other articles were pulled from an independent search for sections on guidelines, UGIB risks, aspirin, clopidogrel, prasugrel, ticagrelor, and PPI pharmacology and interactions.

\section{Guidelines}

The American College of Cardiology (ACC)/American Heart Association (AHA) and European Society of Cardiology (ESC) have published different recommendations for the prescription of DAPT, the duration of treatment, and which agents to prescribe to balance bleeding and ischemic risks.

The 2010 Expert Consensus from the American College of Cardiology Foundation/American College of Gastroenterology/American Heart Association (ACCF/ACG/AHA) stipulates that only patients at high risk of bleeding should receive PPI [7,8]. On the other hand, the 2018 ESC guidelines recommend routine use of PPI for all patients on DAPT [9]. This difference in clinical guidance is largely based on differences in the interpretation of a major clinical trial (COGENT) that demonstrated a pharmacologic interaction between clopidogrel and omeprazole without a clinically significant change in adverse cardiovascular events [7-10]. According to ACCF/ACG/AHA guidelines, patients on DAPT with multiple risk factors for GI bleeding can appropriately be prescribed PPI. The use of PPIs or histamine-2 receptor antagonists (H2RAs) in patients with a low risk of upper GI bleed only showed marginal benefit. Since 2010, no updates have been published to these guidelines [8]. The 2016 ACC/AHA guidelines reiterated this focus on using PPI in only high-risk patients [2].

The ESC recommends that "every effort should be pursued to mitigate the risk of bleeding complications while the patient is on DAPT as appropriate, and routine use of PPI" [9]. The authors cited increased mortality and morbidity associated with bleeding events after successful PCI and, therefore, encouraged the prescription of PPI to all patients on DAPT.

The 2020 International Consensus Recommendations on the Management of Patients With Nonvariceal Upper Gastrointestinal Bleeding state that patients with previous ulcer bleeding receiving cardiovascular prophylaxis with single- or dual-antiplatelet therapy should receive PPI therapy [11]. However, this was a "conditional recommendation" with "low-quality evidence," due to the lack of RCTs. Additionally, these guidelines considered the potential for increased risk of mortality or MI with concurrent DAPT/PPI prescription. Through their own meta-analysis, these authors did not find an increase in mortality from these events in patients receiving DAPT and PPI [11].

The National Association of Hospital Cardiologists (ANMCO) and the Italian Association of Hospital Gastroenterologists (AIGO) recommend the use of PPIs in patients on one antiplatelet agent if GI risk factors are present [12]. Patients receiving antiplatelets and who have a history of peptic ulcer disease (PUD), concomitant use of another antiplatelet agent, concomitant use of vitamin K-antagonists (VKAs) or directacting oral anticoagulants (DOACs), concomitant use of non-steroidal anti-inflammatory drugs (NSAIDs), or concomitant use of steroids should be further considered for PPI use due to increased risk of bleeding. Other risk factors included are age over 65 years, dyspeptic symptoms, and gastroesophageal reflux disease (GERD).

\section{Pharmacology}

DAPT includes two pharmacotherapies: aspirin, an NSAID, and a P2Y12 inhibitor, such as clopidogrel, prasugrel, or ticagrelor. Aspirin has a key role in antiplatelet therapy. Its mechanism of action is the irreversible inhibition of the cyclooxygenase-1 (COX1) enzyme in the arachidonic acid pathway to limit inflammation. For its antiplatelet role, the inhibition of COX1 prevents the conversion of arachidonic acid into prostaglandin $\mathrm{G} 2 / \mathrm{H} 2$ and subsequent reactions by thromboxane synthase into thromboxane A2 (TXA2) [3]. This mechanism makes aspirin effective in preventing serious vascular adverse effects such as MI and stroke. Conversely, aspirin has been infamously associated with an increase in bleeding even when used as monotherapy [3]. By inhibiting COX1, aspirin depletes the protective prostaglandins that promote the integrity of the stomach lining. COX1 inhibition results in decreased mucus secretion, decreased bicarbonate secretion, and mucosal blood flow (Figure 1) [13]. These processes promote UGIB. In a review for the U.S. Preventive Services Task Force (USPSTF), patients who underwent PCI and received low-dose aspirin had a significant increase in major gastrointestinal bleeding risk due to small intestinal mucosal injury $[14,15]$. The addition of DAPT therapy did not increase the risk of aspirin-induced small intestinal mucosal injury, although only 10 patients were in the DAPT group [4]. 


\section{Cureus}

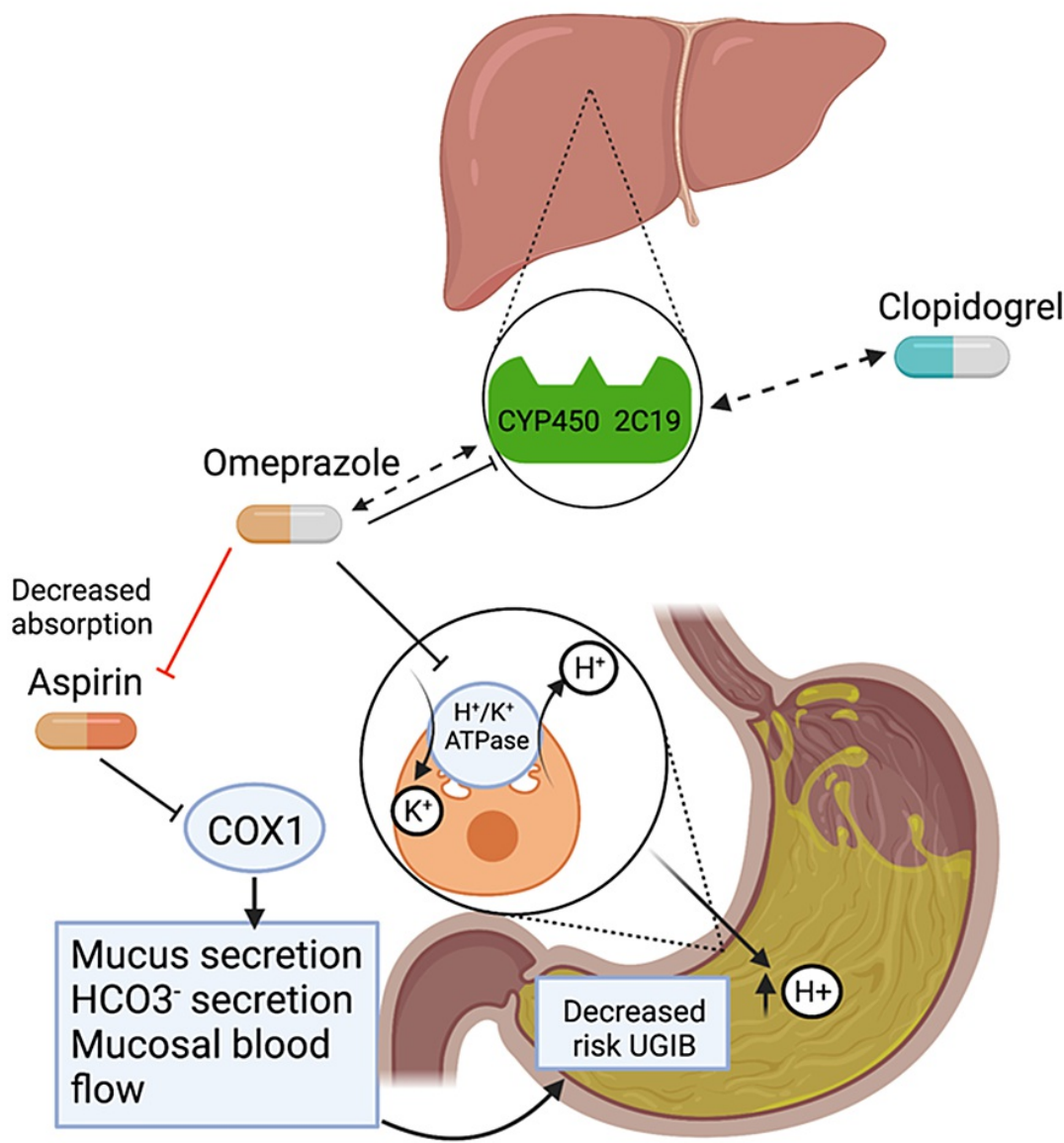

\section{FIGURE 1: Proposed mechanism for DAPT/PPI drug interaction.}

Clopidogrel is metabolized by cytochrome CYP450 2C19. Omeprazole has inhibitory effects and is metabolized by CYP450 2C19, therefore, potentially limiting the active form of clopidogrel and its antiplatelet effects.

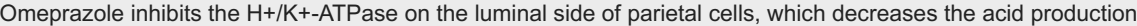
in the stomach. This could limit the absorption of aspirin. On its own, aspirin increases the risk of UGIB by decreasing mucus secretion, decreased bicarbonate secretion, and decreasing mucosal blood flow. The addition of PPI to aspirin typically works to decrease the risk of ulcer formation via decreased acid production. Omeprazole has the potential to affect the absorption and metabolism of aspirin and clopidogrel, respectively. Created with BioRender.com.

DAPT, dual-antiplatelet therapy; PPI, proton pump inhibitor; UGIB, upper gastrointestinal bleed; COX1, cyclooxygenase-1; $\mathrm{H}+/ \mathrm{K}+-$ ATPase, hydrogen potassium adenosine triphosphatase.

Thienopyridines like clopidogrel or prasugrel work by inhibiting the binding of adenosine diphosphate (ADP) to its P2Y12 ADP receptor and requires the transformation by cytochrome P450 enzymes to be activated [16]. In its active form, clopidogrel binds irreversibly to platelet P2Y12 ADP receptor, therefore, inhibiting platelet aggregation. Studies have shown that clopidogrel may have a suboptimal response that can limit its use as an antiplatelet agent in coronary stenting and ACS [17,18]. Prasugrel is more potent than clopidogrel and does not require as many steps in hepatic activation, making it a preferred agent for preventing thrombosis [3]. The main metabolic difference is an enzyme called human carboxylesterase (hCE), which results in a faster onset, greater magnitude, and more consistent levels of platelet inhibition [3]. However, greater potency of the antiplatelet effect also increases bleeding risk. The Greek Antiplatelet (GRAPE) trial showed that prasugrel-treated patients with ACS undergoing had more frequent bleeding than clopidogreltreated patients [19].

Ticagrelor is a cyclopentyltriazolopyrimidine (CPTP) that reversibly binds to the P2Y12 receptor and noncompetitively with ADP [3]. Unlike clopidogrel and prasugrel, ticagrelor is not a prodrug and does not require metabolic activation to exert its effects [3]. Compared to clopidogrel, ticagrelor has also demonstrated greater antiplatelet efficacy [20]. One study showed that ticagrelor had more frequent bleeding compared to clopidogrel-treated patients [19]. Conversely, another study showed that ticagrelor is non-inferior to clopidogrel in both major bleeding events without differences in ischemic events [21]. More data concerning ticagrelor bleeding risks are necessary to provide clinical guidance. 
When DAPT is used, there is a two-fold effect on platelets: aspirin inhibits the production of TXA2 and clopidogrel inhibits P2Y12, both of which inhibit platelet activation and platelet aggregation. P2Y12 receptor inhibitors are not directly responsible for creating ulcers in the gastric lining as aspirin is, but its antiplatelet activity confers a potential risk for bleeding.

PPIs directly stop gastric acid secretion by irreversibly binding to hydrogen potassium adenosine triphosphatase (H+/K+-ATPase) on the luminal membrane of parietal cells in the lining of the stomach (Figure 1). This group of medications includes omeprazole, lansoprazole, pantoprazole, rabeprazole, esomeprazole, and dexlansoprazole. PPIs are metabolized by and competitively inhibit CYP2C19 (Figure 1) and CYP3A4 pathways except for pantoprazole, lansoprazole, rabeprazole, and dexlansoprazole. According to the American College of Gastroenterology (ACG), the PPI indications include PUD, GERD, erosive esophagitis, and hypersecretory pathologies like Zollinger-Ellison syndrome [22]. PPIs may also be used to prevent stress ulcers in ICU settings and prevent the formation of ulcers from NSAID use. Full adherence to PPIs was the only gastroprotective approach that significantly reduced the risk of UGIB when taking NSAIDs even at low doses, with even greater risk at higher dose NSAIDs [23]. The same effect could not be achieved with histamine 2 receptor antagonists (H2RAs). While PPIs block $\mathrm{H}^{+} / \mathrm{K}+-$ ATPase on the luminal face of the parietal cells, H2RAs indirectly inhibit acid secretion by binding to the histamine 2 receptor on the basolateral side of the parietal cell. This receptor works in conjunction with acetylcholine and gastrin receptors to promote acid secretion from the parietal cell. However, there is varied evidence as to whether H2RAs are effective in preventing NSAID-induced bleeding or DAPT-induced bleeding. One study showed that long-term PPI use reduced the risk of UGIB recurrence, but this effect was not observed with patients on H2RAs [24]. On the opposite side, no significant GI bleeding was reported in patients at risk for recurrent GI bleeding who were on low-dose aspirin regardless of if they had received rabeprazole [25]. Furthermore, other studies proposed that H2RAs prevented low-dose aspirin-related GI bleeding and ulcer formation significantly less than PPI [26,27].

Over the last few years, clinicians have been wary of prescribing PPI due to observational studies proposing correlations with adverse effects like chronic kidney disease (CKD) and dementia [12]. The ACG acknowledges side effects of PPIs have been reported in retrospective observational studies [22]. However, no RCTs have been performed to assess the validity of these adverse effects [28]. Low-quality evidence should not be used to guide clinicians and withhold PPIs from patients who might benefit from its use [29].

\section{Epidemiology: who is at risk of bleeding?}

DAPT can put certain patient groups at increased risk of bleeding. ACCF/ACG/AHA currently recommends PPI prophylaxis for patients on DAPT who have Helicobacter pylori infection, advanced age, or use of warfarin, steroids, or NSAIDs [8]. In these individuals, the risk of bleeding outweighs the risk of thrombosis. Other risk factors for an upper GI bleed identified by this study include female sex, major organs dysfunction, hemostatic disorders, or a history of hemorrhage [30].

Older patients tend to develop disabling or fatal UGIBs compared to younger patients [31]. Younger individuals were more likely to have non-disabling UGIB with lower case fatality [3]. Older patients who are at higher risk for UGIB had decreased mortality when prescribed PPI along with DAPT. However, the ceiling effect could also account for an imprecise number needed to treat (NNT) for PPI in individuals aged >85 years. Five-year risk of bleeding can exceed the life expectancy of elderly patients, resulting in artificial inflating NNT [32]. In addition to advanced age and female gender, $H$. pylori infection, history of digestive tract disease, diabetes, hypertension, dyslipidemia, anemia, CKD, peripheral vascular disease, and smoking have been recognized as risk factors for increased risk of GI bleed [33]. Patients in the high-risk bleeding group were also more likely to have had a previous MI, previous PCI with stent implantation, surgical revascularization, and stroke [34].

\section{Who is getting a DAPT/PPI prescription?}

Since 2010, concurrent use of clopidogrel and PPI has steadily decreased due to the 2009 FDA warning that omeprazole and esomeprazole could limit the antiplatelet activity of clopidogrel [35]. This finding led to the updating of clopidogrel's label noting its interaction with omeprazole. Non-inhibiting PPIs such as pantoprazole, lansoprazole, rabeprazole, and dexlansoprazole prescription rates were not affected by this warning. In 2016, the prevalence of clopidogrel and inhibiting PPIs among ACS patients was $0.8 \%$ compared to $34.9 \%$ in 2009 . This study also found that younger inpatients were less likely to receive concurrent treatment compared to older groups, defined as 65 years or older.

Although European guidelines recommend all patients receiving DAPT should take PPI, similar rates of coprescription are seen between European countries and the United States. When considering ESC guidelines, PPI prescribing trends in patients taking DAPT seven days following hospital discharge for an acute MI did not meet ESC criteria that recommended PPI prescription for all patients on DAPT regardless of upper GI risk factors $[9,36]$. Only $35 \%$ of patients with a higher risk of UGIB received recommended PPI treatment [36]. Overall, prescribing trends are much lower than recommended. This observation is reflective of the overall skepticism of prescribing PPI to patients on DAPT despite guidelines. 


\section{DAPT increases bleeding risk}

DAPT increases bleeding risk because of the pharmacological interaction with platelet function. Dewilde et al. (2013) studied bleeding complications on PCI patients who used oral anticoagulation (AC) and clopidogrel with or without aspirin [37]. AC increases the risk of bleeding. Patients receiving double therapy (AC and clopidogrel) had fewer bleeding episodes than those on triple therapy (AC, clopidogrel, and aspirin) [37]. Clopidogrel monotherapy in PCI patients was associated with a significantly lower rate of bleeding complications compared to dual therapy (clopidogrel and aspirin). Importantly, there were no additional thrombotic events in the monotherapy group. Adding aspirin to clopidogrel in PCI patients results in an increased bleeding risk [38-40]. Some of these studies suggest aspirin is the component of DAPT that increases the risk for GI bleeding [38,39]. One study has shown a two- to three-fold increased risk of gastrointestinal bleeding and another major bleeding in DAPT compared to monotherapies [39]. However, low-dose aspirin had a better safety profile compared to other pharmaceuticals [39]. In the same study, clopidogrel was associated with a non-significant increased risk of major bleeding events and was found significantly better in secondary prevention than low-dose aspirin in the prevention of event-free survival.

\section{Notable interactions}

Aspirin/PPI Interaction

In the context of DAPT, controversy remains as to whether concurrent use of PPI is more harmful or helpful. The acidic nature of the stomach facilitates the absorption of aspirin; therefore, the administration of PPIs decreases the acid secretion and increases gastric $\mathrm{pH}$, which can potentially interfere with aspirin absorption (Figure 1). A case-control study of CAD patients treated with and without PPIs found that platelet aggregation and activation were significantly increased in the patients receiving PPI, increasing the chances of thrombotic events [41]. However, the study does not elucidate the causality of PPI treatment to aspirin response. On the other hand, other researchers argue that concurrent PPI and aspirin use could prevent upper GI toxicity from aspirin therapy, and coadministration would increase adherence to aspirin therapy [42]. Compared to nonuse of PPIs, continuous PPI use was associated with a lower risk of aspirin discontinuation among patients with a high risk for upper GI events [43].

Clopidogrel/PPI

Due to clopidogrel's metabolism by CYP450 liver enzymes and some PPIs inhibition of CYP450, concurrent use of clopidogrel and PPIs could decrease the overall efficacy of clopidogrel (Figure 1). A study by Juurlink et al. (2009) was one of the first studies to describe this interaction via the CYP450 pathway [44]. In this study of patients following MI, researchers found that the use of PPIs with clopidogrel was associated with a $40 \%$ increase in the risk of recurrent MI within 90 days of hospital discharge [44]. However, pantoprazole, which does not inhibit CYP450 2C19, was not associated with readmissions for myocardial infarction.

In contrast, another study demonstrated that PPI and clopidogrel use in patients after ischemic stroke was not associated with recurrent ischemic stroke, MI, intracerebral hemorrhage, and death [45]. These authors concluded that PPIs did not affect platelet aggregation in patients taking clopidogrel. More research is needed to investigate the proposed CYP450-related interactions between concomitant PPI and clopidogrel therapy.

\section{Prasugrel/PPI and Ticagrelor/PPI}

With newer P2Y12 inhibitors earning favor over clopidogrel, the use of PPIs may become even more important because these newer agents are more potent. The stronger antiplatelet effect of prasugrel can lead to renewed bleeding concerns. There is less literature concerning interactions between PPIs and prasugrel or ticagrelor. Like clopidogrel, prasugrel is still metabolized by CYP2C19 into its active form [12]. One study found that patients on prasugrel and PPI were less likely to have MI than patients on clopidogrel and PPI [46]. However, this study had a surrogate endpoint, which led to skepticism over the increased observed cardiovascular risk [12]. One position paper from Italy stated that if a patient is on clopidogrel, then rabeprazole or pantoprazole should be the PPI of choice if patients have at least one risk factor [47]. If a patient is on prasugrel or ticagrelor with at least one GI bleeding risk factor, any choice of PPI may be used [47]. Despite these recommendations, there is little evidence to support interaction or lack thereof between prasugrel and PPIs. Conversely, another study found that PPI use in patients did not significantly affect the comparative effectiveness or bleeding risk of prasugrel compared to clopidogrel [48].

In theory, ticagrelor should not hepatically interact with PPIs since it is not a prodrug and does not need to be metabolized. Its stronger potency may lead to increased bleeding risks, though this risk has yet to be thoroughly studied [19-21]. In terms of interactions with PPI, one study that looked at concomitant use of PPI and clopidogrel or ticagrelor in patients with ACS did not find any significant differences between allcause death, re-infarction, or decreased risk of severe bleeding [49]. A separate study substantiated the lack of difference in death, MI, and GI bleeding between ACS patients treated with clopidogrel or ticagrelor and PPIs [50]. While the current literature is limited, the results indicate that these separate agents do not change major outcomes when taken with PPI and that these results are like those of patients that took 


\section{Use of PPI while on DAPT}

While co-prescription of PPI alongside DAPT is associated with decreased risk of UGIB, it has also been associated with decreased efficacy of clopidogrel in preventing thrombosis. Only one RCT to date has studied co-therapy of DAPT and omeprazole [10]. In the COGENT, patients taking DAPT and omeprazole had significantly less overt or occult bleeding, symptomatic gastroduodenal ulcers or erosions, and obstruction or perforation than patients taking DAPT with no PPIs. Patients taking esomeprazole also had decreased rate of overt UGIB (HR, 0.13; 95\% CI, 0.03-0.56; $\mathrm{P}=0.001$ ). There were no significant differences between omeprazole and placebo groups in deaths from cardiovascular causes, nonfatal myocardial infarction, revascularization, or stroke (HR with omeprazole, 0.99; 95\% CI, 0.68-1.44; $\mathrm{P}=0.96$ ). The study controlled for NSAID use and $H$. pylori infection. Unfortunately, the trial lost sponsor financing and was terminated early. Unlike previous observational studies, this RCT concluded that DAPT and PPI, specifically clopidogrel and omeprazole, did not increase the risk for cardiovascular adverse events. The study was limited by decreased power as fewer adverse events occurred than anticipated. Additionally, COGENT excluded patients with prior indications for PPIs/H2RAs as well as patients with upper GI pathologies and previous AC or fibrinolytic therapy. The generalizability of the results of the COGENT study may also be limited as $94 \%$ of study participants were White and only one PPI, omeprazole, was used. According to COGENT results, the benefit of DAPT and PPI co-therapy (decreased GI bleeding) outweighed the potential risks (adverse cardiovascular events due to decreased DAPT efficacy).

Post hoc analysis of COGENT patients [51] found that among PCI-treated patients, omeprazole significantly reduced rates of composite gastrointestinal events without increasing composite cardiovascular events. Similarly, in patients with ACS, omeprazole lowered the risk of primary gastrointestinal events compared to placebo without a significant increase in cardiovascular events. This post hoc analysis in high-risk cardiovascular patients supports the use of clopidogrel and PPIs without increasing the risk for cardiovascular complications.

In another post hoc analysis, high-dose and low-dose aspirin had similar risks of gastrointestinal events and major adverse cardiac events aspirin [52]. The addition of PPI therapy led to a reduction of primary GI endpoint in both subsets. These various analyses of COGENT highlight the safety of concurrent PPIs with DAPT therapy and further support its efficacy in decreasing the incidence of upper GI bleeding without major side effects.

Additional studies have corroborated the finding that PPIs reduce the risk of UGIB, GI ulcers, and erosions in patients on concurrent DAPT [36,53-56]. Screening for risk factors of upper GI bleeding did not significantly reduce the incidence of UGIB in patients placed on DAPT and PPI [57]. The ACCF/AGA/AHA only recommends PPIs concurrently with DAPT in groups with bleeding risk factors [8]; the benefit of PPIs and DAPT therapy outweigh any potential risks, but more research is needed to determine whether this benefit may only apply to patients in a high-risk group [36,57].

There is evidence beyond COGENT data that PPI and DAPT co-therapy does not lead to an increase in MACCE. There are substantial data that demonstrate that patients treated with PCI and DAPT (clopidogrel and aspirin) have had similar all-cause death, MI, and cerebrovascular accident (CVA) among PPI and nonPPI users [27,53-60]. A couple of studies in patients with CAD also concluded PPI and DAPT co-prescription did not lead to increased adverse risks [56,60]. Patients screened for UGIB risk factors such as old age, dyspepsia, previous uncomplicated ulcer, previous ulcer bleeding, NSAID, corticosteroid, selective serotonin reuptake inhibitor (SSRI), or oral anticoagulant on DAPT/PPI therapy had significantly fewer events of unstable angina pectoris compared to controls [57]. This development is in line with ACCF/ACG/AHA guidelines [8]. Compared to COGENT, the Prolonging Dual-antiplatelet Treatment after Grading Stentinduced Intimal Hyperplasia Study (PRODIGY) included patients with a higher risk of bleeding or patients that had other indications for PPIs [57]. The patients on PPIs were more likely to be older, female, have lower creatinine clearance, higher bleeding score, and present more frequently with ACS [58]. Despite these increased risk factors for bleeding, patients on PPIs/DAPT and DAPT had similar rates of all-cause death, MI, and CVA. Though these studies point to a favorable safety profile between DAPT and PPI, one study pointed out a higher risk of ischemic stroke and MI observed in long-term PPI use [36]. This concern has already been addressed by the ACG [22]. In the last five years, there has been a myriad of data establishing that PPI does not decrease the efficacy of DAPT.

Some studies propose that the use of PPIs with DAPT may in fact decrease MACCE risk [61]. Patients screened for UGIB risk and prophylactically treated with pantoprazole had increased compliance to DAPT [57,61]. This mirrors the mechanism proposed by the aspirin/PPI co-administration [42]. Patients who were screened and given PPI prophylaxis had higher compliance with treatment of an ADP-receptor inhibitor like clopidogrel [57]. The researchers predicted that dyspepsia related to antiplatelet therapy was reduced by taking PPIs. The use of PPIs to not only prevent DAPT-related bleeding but also to treat dyspepsia symptoms caused by DAPT could be a novel prospect [43].

Potential racial and ethnic differences should also be considered. One meta-analysis/systematic review of 
observational studies [62] showed that among Caucasian populations, DAPT and PPI concurrent use was associated with an increased risk for MACCE, all-cause death, and all other studied clinical adverse events. However, in Asian populations, no significant differences in MACCE, all-cause death, cardiac death, and stroke were found between PPI and non-PPI users. Without racial stratification, the results of this study did not show any benefit in the concurrent prescription of PPIs and DAPT [62]. This new perspective could elucidate why studies offer contradictory findings of DAPT and PPI concurrent use. When studying stent implantation, one study found that MACCE was increased in PPI users due to increased stent thrombosis, without differences in death, ST, or MI compared with non-PPI users [63]. However, these differences were driven by underlying confounding patient characteristics in the PPI group such as higher incidence of bifurcation lesions, bare-metal stents, and higher risk of target lesion revascularization rather than increased atherothrombotic risk [63]. Study limitations include observational nature, physician discretion for PPI prescription, lack of PPI compliance follow-up, and patient-reported DAPT adherence [63]. Additionally, DAPT compliance was self-reported.

\section{Discussion}

The concurrent use of DAPT and PPIs has continued to be debated since COGENT results were published in 2010. Despite its loss of funding and early termination, COGENT had enough data to show that concurrent therapy with PPI and DAPT led to a significant reduction of upper GI bleeds, erosions, and ulcers without increasing adverse events. However, subsequent observational studies suggested that PPIs and P2Y12 inhibitors were associated with increased major cardiac adverse events, via CYP450 inhibition, demonstrating statistically significant results $(\mathrm{p}=0.05)$ with an OR of 1.27 for reinfarction and 1.4 for risk of myocardial infarction [44].

As COGENT has been the only large, multi-center, international, double-blind, phase 3 RCT in this topic, there is a paucity of data needed to yield strong recommendations on DAPT and PPI co-therapy. In the past five years, the academic milieu has been flooded with studies providing murky evidence for proposing PPI protection for GI bleed for patients on DAPT therapy or arguing PPIs decrease the efficacy of DAPT therapy, therefore, increasing the risk of cardiovascular adverse events or stent thrombosis. To build upon COGENT, future studies should include stratification of medications with and without CYP450 inhibition concurrently with DAPT. While there have been studies that look at how UGIB screening risk affects the incidence of bleeding [57], no RCT study has investigated the effects of PPI and DAPT in patients with a history of ulcer bleeding. Additionally, since the COGENT study ended early, new studies should also aim to examine any long-term effects of concurrent therapy.

Another factor to consider is potential racial and ethnic differences; concurrent PPI and DAPT have been studied in majority Caucasian and Asian populations [62]. The authors propose that patients with a hypofunctional CYP2C19 metabolic phenotype may benefit from concurrent DAPT/PPI therapy [62]. Racial and ethnic differences could contribute to the variation in study findings of DAPT and PPI concurrent use. More research is needed to further investigate this mechanism and could potentially provide more personalized care to patients.

Current American clinical guidelines tend to value conservative management, recommending PPI coprescription with DAPT only in patients with a high risk of bleeding [8]. As it stands, there is not enough evidence to recommend PPI to all patients receiving DAPT [8]. However, the benefits of concurrent DAPT/PPI prescription are not limited to a decrease in UGIB risk. For example, some studies have shown patients on DAPT and PPIs could benefit from increased medication compliance to DAPT [57].

Future studies should also consider the use of third-generation P2Y12 inhibitors such as ticagrelor. Most studies only considered clopidogrel as the antiplatelet agent for DAPT. COGENT only examines the effects of clopidogrel. Comparing the efficacy of these newer agents concurrently with PPIs could provide meaningful data about the best combination of drugs that maximizes efficacy and decreases the risk of adverse effects. For example, ticagrelor, unlike clopidogrel, does not need hepatic enzyme activation $[3,64]$. Therefore, this could be a viable solution for clinicians and patients concerned with both gastrointestinal bleeding and potential interactions between clopidogrel and a PPI. The current literature on the use of newer, alternate P2Y12 and PPIs is quite limited, and more research is needed to determine if there is any interaction between PPIs and ticagrelor or prasugrel, or to determine if there is any benefit in using these agents concurrently with PPIs instead of clopidogrel. These data will be important especially since ticagrelor and prasugrel are becoming preferred agents over clopidogrel, which has had inconsistent antiplatelet responses $[17,18]$. Therefore, this could be a viable solution for clinicians and patients concerned with both gastrointestinal bleeding and potential interactions between clopidogrel and a PPI. For many of these studies, particularly the observational studies, the choice of both PPI and P2Y12 inhibitors was left to individual physician discretion.

A new meta-analysis reviewing RCTs of DAPT following PCI showed that DAPT trials after PCI often overlook gastroprotection entirely [65]. Among 21 trials, none incorporated any protocol for PPI or gastroprotection. However, five trials reported rates of PPI use (25.6-69.1\%). Underutilization of gastroprotection was also reported in another study [66]. Trials utilizing DAPT in the future could provide clear guidelines on PPI use and strengthen the power of meta-analyses. 


\section{Conclusions}

Guidelines vary internationally recommending the concurrent use of PPI and DAPT. The 2010 ACCF/ACG/AHA guidelines recommend that only patients on DAPT with increased risk of UGIB should take PPI, while other guidelines call for gastroprotection for all patients on DAPT. Both aspirin and clopidogrel can interact with PPI, potentially decreasing their antiplatelet effects. However, the evidence for this is not particularly strong. COGENT was the only large RCT investigating patients on concurrent DAPT and PPI therapy. There have been smaller RCTs, various meta-analyses, and observational studies that have proposed conflicting evidence of this regimen's efficacy. While some studies still warn of increased MACCE from concurrent therapy, other studies have shown beneficial effects such as a decrease in UGIB. Another large-scale RCT is needed to better understand the short- and long-term effects of concurrent DAPT and PPI use. Future studies should consider how dyspepsia symptoms affect treatment adherence, account for potential racial and ethnic differences, and account for baseline risk levels for related adverse events. Altogether, a more intensive study of DAPT/PPI co-prescription could provide patients with improved personalized care that meets their specific symptoms and accounts for their particular risk levels, with the goal of improving overall health outcomes.

\section{Additional Information \\ Disclosures}

Conflicts of interest: In compliance with the ICMJE uniform disclosure form, all authors declare the following: Payment/services info: All authors have declared that no financial support was received from any organization for the submitted work. Financial relationships: All authors have declared that they have no financial relationships at present or within the previous three years with any organizations that might have an interest in the submitted work. Other relationships: All authors have declared that there are no other relationships or activities that could appear to have influenced the submitted work.

\section{Acknowledgements}

The figure included in this article was created with BioRender.com.

\section{References}

1. Capodanno D, Alfonso F, Levine GN, Valgimigli M, Angiolillo DJ: ACC/AHA versus ESC guidelines on dual antiplatelet therapy: JACC guideline comparison. J Am Coll Cardiol. 2018, 72:2915-31. 10.1016/j.jacc.2018.09.057

2. Levine GN, Bates ER, Bittl JA, et al.: 2016 ACC/AHA guideline focused update on duration of dual antiplatelet therapy in patients with coronary artery disease: a report of the American College of Cardiology/American Heart Association Task Force on Clinical Practice Guidelines. J Am Coll Cardiol. 2016, 68:1082-115. 10.1016/j.jacc.2016.03.513

3. Angiolillo DJ: The evolution of antiplatelet therapy in the treatment of acute coronary syndromes: from aspirin to the present day. Drugs. 2012, 72:2087-116. 10.2165/11640880-000000000-00000

4. Airoldi F, Colombo A, Morici N, et al.: Incidence and predictors of drug-eluting stent thrombosis during and after discontinuation of thienopyridine treatment. Circulation. 2007, 116:745-54. 10.1161/CIRCULATIONAHA.106.686048

5. Kuchulakanti PK, Chu WW, Torguson R, et al.: Correlates and long-term outcomes of angiographically proven stent thrombosis with sirolimus- and paclitaxel-eluting stents. Circulation. 2006, 113:1108-13. 10.1161/CIRCULATIONAHA.105.600155

6. Costa F, Van Klaveren D, Feres F, et al.: Dual antiplatelet therapy duration based on ischemic and bleeding risks after coronary stenting. J Am Coll Cardiol. 2019, 73:741-54. 10.1016/j.jacc.2018.11.048

7. Floyd CN: Dual antiplatelet therapy in coronary artery disease: comparison between ACC/AHA 2016 and ESC 2017 guidelines. Eur Cardiol. 2020, 15:1-3. 10.15420/ecr.2019.09

8. Abraham NS, Hlatky MA, Antman EM, et al.: ACCF/ACG/AHA 2010 expert consensus document on the concomitant use of proton pump inhibitors and thienopyridines: a focused update of the ACCF/ACG/AHA 2008 expert consensus document on reducing the gastrointestinal risks of antiplatelet therapy and NSAID use: a report of the American College of Cardiology Foundation Task Force on Expert Consensus Documents. Circulation. 2010, 122:2619-33. 10.1161/CIR.0b013e318202f701

9. Valgimigli M, Bueno H, Byrne RA, et al.: 2017 ESC focused update on dual antiplatelet therapy in coronary artery disease developed in collaboration with EACTS: the Task Force for Dual Antiplatelet Therapy in Coronary Artery Disease of the European Society of Cardiology (ESC) and of the European Association for Cardio-Thoracic Surgery (EACTS). Eur Heart J. 2018, 39:213-60. 10.1093/eurheartj/ehx419

10. Bhatt DL, Cryer BL, Contant CF, et al.: Clopidogrel with or without omeprazole in coronary artery disease. N Engl J Med. 2010, 363:1909-17. 10.1056/NEJMoa1007964

11. Barkun AN, Almadi M, Kuipers EJ, et al.: Management of nonvariceal upper gastrointestinal bleeding: guideline recommendations from the International Consensus Group. Ann Intern Med. 2019, 171:805-22. 10.7326/M19-1795

12. Abrignani MG, Gatta L, Gabrielli D, et al.: Gastroprotection in patients on antiplatelet and/or anticoagulant therapy: a position paper of National Association of Hospital Cardiologists (ANMCO) and the Italian Association of Hospital Gastroenterologists and Endoscopists (AIGO). Eur J Intern Med. 2021, 85:1-13. 10.1016/j.ejim.2020.11.014

13. Iwamoto J, Saito Y, Honda A, Matsuzaki Y: Clinical features of gastroduodenal injury associated with longterm low-dose aspirin therapy. World J Gastroenterol. 2013, 19:1673-82. 10.3748/wjg.v19.i11.1673 
14. Whitlock EP, Burda BU, Williams SB, Guirguis-Blake JM, Evans CV: Bleeding risks with aspirin use for primary prevention in adults: a systematic review for the U.S. Preventive Services Task Force. Ann Intern Med. 2016, 164:826-35. 10.7326/M15-2112

15. Hara A, Ota K, Takeuchi T, et al.: Dual antiplatelet therapy does not affect the incidence of low-dose aspirininduced small intestinal mucosal injury in patients after percutaneous coronary intervention for coronary stenosis: a multicenter cross-sectional study. J Clin Biochem Nutr. 2018, 63:224-9. 10.3164/jcbn.18-16

16. Bolek T, Samoš M, Škorňová I, et al.: Proton pump inhibition in patients treated with novel antithrombotic drugs: should we worry about thrombosis? J Cardiovasc Pharmacol. 2018, 72:71-6. 10.1097/FJC.0000000000000593

17. Bonello L, Armero S, Ait Mokhtar O, et al.: Clopidogrel loading dose adjustment according to platelet reactivity monitoring in patients carrying the $2 \mathrm{C} 19 * 2$ loss of function polymorphism. J Am Coll Cardiol. 2010, 56:1630-6. 10.1016/j.jacc.2010.07.004

18. Gurbel PA, Bliden KP, Hiatt BL, O'Connor CM: Clopidogrel for coronary stenting: response variability, drug resistance, and the effect of pretreatment platelet reactivity. Circulation. 2003, 107:2908-13. 10.1161/01.CIR.0000072771.11429.83

19. Alexopoulos D, Xanthopoulou I, Deftereos S, et al.: Contemporary antiplatelet treatment in acute coronary syndrome patients undergoing percutaneous coronary intervention: 1-year outcomes from the GReek AntiPlatElet (GRAPE) Registry. J Thromb Haemost. 2016, 14:1146-54. 10.1111/jth.13316

20. Husted S, Emanuelsson H, Heptinstall S, Sandset PM, Wickens M, Peters G: Pharmacodynamics, pharmacokinetics, and safety of the oral reversible P2Y12 antagonist AZD6140 with aspirin in patients with atherosclerosis: a double-blind comparison to clopidogrel with aspirin. Eur Heart J. 2006, 27:1038-47. 10.1093/eurheartj/ehi754

21. Berwanger O, Nicolau JC, Carvalho AC, et al.: Ticagrelor vs clopidogrel after fibrinolytic therapy in patients with ST-elevation myocardial infarction: a randomized clinical trial. JAMA Cardiol. 2018, 3:391-9. 10.1001/jamacardio.2018.0612

22. Laine L, Nagar A: Long-term PPI use: balancing potential harms and documented benefits . Am J Gastroenterol. 2016, 111:913-5. 10.1038/ajg.2016.156

23. Ruiz B, Aguirre U, Estany-Gestal A, et al.: Only full adherence to proton pump inhibitors protects against drug-induced upper gastrointestinal bleeding. Eur J Clin Pharmacol. 2018, 74:1503-11. 10.1007/s00228-0182523-4

24. Massó González EL, García Rodríguez LA: Proton pump inhibitors reduce the long-term risk of recurrent upper gastrointestinal bleeding: an observational study. Aliment Pharmacol Ther. 2008, 28:629-37. 10.1111/j.1365-2036.2008.03780.x

25. Chan FK, Kyaw M, Tanigawa T, et al.: Similar efficacy of proton-pump inhibitors vs H2-receptor antagonists in reducing risk of upper gastrointestinal bleeding or ulcers in high-risk users of low-dose aspirin. Gastroenterology. 2017, 152:105-10. 10.1053/j.gastro.2016.09.006

26. Szabó IL, Mátics R, Hegyi P, et al.: PPIs prevent aspirin-induced gastrointestinal bleeding better than H2RAs. A systematic review and meta-analysis. J Gastrointestin Liver Dis. 2017, 26:395-402. 10.15403/igld.2014.1121.264.hra

27. Almufleh A, Ramirez FD, So D, Le May M, Chong AY, Torabi N, Hibbert B: H2 receptor antagonists versus proton pump inhibitors in patients on dual antiplatelet therapy for coronary artery disease: a systematic review. Cardiology. 2018, 140:115-23. 10.1159/000489165

28. Manolis AA, Manolis TA, Melita H, Katsiki N, Manolis AS: Proton pump inhibitors and cardiovascular adverse effects: real or surreal worries?. Eur J Intern Med. 2020, 72:15-26. 10.1016/j.ejim.2019.11.017

29. Schubert ML: Proton pump inhibitors: placing putative adverse effects in proper perspective . Curr Opin Gastroenterol. 2019, 35:509-16. 10.1097/MOG.0000000000000580

30. Yasuda H, Matsuo Y, Sato Y, et al.: Treatment and prevention of gastrointestinal bleeding in patients receiving antiplatelet therapy. World J Crit Care Med. 2015, 4:40-6. 10.5492/wjccm.v4.i1.40

31. Li L, Geraghty OC, Mehta Z, Rothwell PM: Age-specific risks, severity, time course, and outcome of bleeding on long-term antiplatelet treatment after vascular events: a population-based cohort study. Lancet. 2017, 390:490-9. 10.1016/S0140-6736(17)30770-5

32. Porta L, Baorda F, Fusco A: Is proton pump inhibitors' prophylaxis indicated for patients on antiplatelet therapy?. Intern Emerg Med. 2018, 13:585-7. 10.1007/s11739-018-1827-9

33. Sorrentino S, Sartori S, Baber U, et al.: Bleeding risk, dual antiplatelet therapy cessation, and adverse events after percutaneous coronary intervention: the PARIS Registry. Circ Cardiovasc Interv. 2020, 13:e008226. 10.1161/CIRCINTERVENTIONS.119.008226

34. Tang B, Xiao S: Logistic regression analysis of risk factors for upper gastrointestinal bleeding induced by PCI in combination with double antiplatelet therapy for STEMI patients. Acta Gastroenterol Belg. 2020, 83:2458.

35. Farhat N, Haddad N, Crispo J, et al.: Trends in concomitant clopidogrel and proton pump inhibitor treatment among ACS inpatients, 2000-2016. Eur J Clin Pharmacol. 2019, 75:227-35. 10.1007/s00228-0182564-8

36. Sehested TS, Carlson N, Hansen PW, et al.: Reduced risk of gastrointestinal bleeding associated with proton pump inhibitor therapy in patients treated with dual antiplatelet therapy after myocardial infarction. Eur Heart J. 2019, 40:1963-70. 10.1093/eurheartj/ehz104

37. Dewilde WJ, Oirbans T, Verheugt FW, et al.: Use of clopidogrel with or without aspirin in patients taking oral anticoagulant therapy and undergoing percutaneous coronary intervention: an open-label, randomised, controlled trial. Lancet. 2013, 381:1107-15. 10.1016/S0140-6736(12)62177-1

38. García Rodríguez LA, Martín-Pérez M, Hennekens CH, Rothwell PM, Lanas A: Bleeding risk with long-term low-dose aspirin: a systematic review of observational studies. PLoS One. 2016, 11:e0160046. 10.1371/journal.pone.0160046

39. Bouget J, Balusson F, Viglino D, Roy PM, Lacut K, Pavageau L, Oger E: Major bleeding risk and mortality associated with antiplatelet drugs in real-world clinical practice. A prospective cohort study. PLoS One. 2020, 15:e0237022. 10.1371/journal.pone.0237022 
40. Sugisaki N, Iwakiri R, Tsuruoka N, et al.: A case-control study of the risk of upper gastrointestinal mucosal injuries in patients prescribed concurrent NSAIDs and antithrombotic drugs based on data from the Japanese national claims database of 13 million accumulated patients. J Gastroenterol. 2018, 53:1253-60. 10.1007/s00535-018-1483-X

41. Würtz M, Grove EL, Kristensen SD, Hvas AM: The antiplatelet effect of aspirin is reduced by proton pump inhibitors in patients with coronary artery disease. Heart. 2010, 96:368-71. 10.1136/hrt.2009.181107

42. Lavie CJ, Howden CW, Scheiman J, Tursi J: Upper gastrointestinal toxicity associated with long-term aspirin therapy: consequences and prevention. Curr Probl Cardiol. 2017, 42:146-64. 10.1016/j.cpcardiol.2017.01.006

43. Martín Merino E, Johansson S, Nagy P, García Rodríguez LA: Effect of baseline gastrointestinal risk and use of proton pump inhibitors on frequency of discontinuation of aspirin for secondary cardiovascular prevention in United Kingdom primary care. Am J Cardiol. 2013, 112:1075-82. 10.1016/j.amjcard.2013.05.051

44. Juurlink DN, Gomes T, Ko DT, et al.: A population-based study of the drug interaction between proton pump inhibitors and clopidogrel. CMAJ. 2009, 180:713-8. 10.1503/cmaj.082001

45. Yi X, Zhou Q, Wang C, Lin J, Cheng W, Chi L: Concomitant use of proton pump inhibitors and clopidogrel is not associated with adverse outcomes after ischemic stroke in Chinese population. J Stroke Cerebrovasc Dis. 2016, 25:2859-67. 10.1016/j.jstrokecerebrovasdis.2016.08.001

46. Nicolau JC, Bhatt DL, Roe MT, et al.: Concomitant proton-pump inhibitor use, platelet activity, and clinical outcomes in patients with acute coronary syndromes treated with prasugrel versus clopidogrel and managed without revascularization: insights from the targeted platelet inhibition to clarify the optimal strategy to medically manage acute coronary syndromes trial. Am Heart J. 2015, 170:683-94. 10.1016/j.ahj.2015.05.017

47. Scarpignato C, Gatta L, Zullo A, Blandizzi C: Effective and safe proton pump inhibitor therapy in acid-related diseases - a position paper addressing benefits and potential harms of acid suppression. BMC Med. 2016, 14:179. 10.1186/s12916-016-0718-z

48. Jackson LR 2nd, Peterson ED, McCoy LA, et al.: Impact of proton pump inhibitor use on the comparative effectiveness and safety of prasugrel versus clopidogrel: insights from the treatment with adenosine diphosphate receptor inhibitors: longitudinal assessment of treatment patterns and events after acute coronary syndrome (TRANSLATE-ACS) study. J Am Heart Assoc. 2016, 5:e003824. 10.1161/JAHA.116.003824

49. Yan Y, Wang X, Fan JY, et al.: Impact of concomitant use of proton pump inhibitors and clopidogrel or ticagrelor on clinical outcomes in patients with acute coronary syndrome. J Geriatr Cardiol. 2016, 13:209-17. 10.11909/j.issn.1671-5411.2016.03.007

50. Hoedemaker NP, Damman P, Ottervanger JP, et al.: Trends in cardiovascular and bleeding outcomes in acute coronary syndrome patients treated with or without proton-pump inhibitors during the introduction of novel P2Y12 inhibitors: a five-year experience from a single-centre observational registry. Eur Heart J Cardiovasc Pharmacother. 2019, 5:127-38. 10.1093/ehjcvp/pvy030

51. Vaduganathan M, Cannon CP, Cryer BL, et al.: Efficacy and safety of proton-pump inhibitors in high-risk cardiovascular subsets of the COGENT trial. Am J Med. 2016, 129:1002-5. 10.1016/j.amjmed.2016.03.042

52. Vaduganathan M, Bhatt DL, Cryer BL, et al.: Proton-pump inhibitors reduce gastrointestinal events regardless of aspirin dose in patients requiring dual antiplatelet therapy. J Am Coll Cardiol. 2016, 67:166171. 10.1016/j.jacc.2015.12.068

53. Khan MY, Siddiqui WJ, Alvarez C, Aggarwal S, Hasni SF, Ahmad A, Eisen H: Reduction in postpercutaneous coronary intervention angina in addition to gastrointestinal events in patients on combined proton pump inhibitors and dual antiplatelet therapy: a systematic review and meta-analysis. Eur J Gastroenterol Hepatol. 2018, 30:847-53. 10.1097/MEG.0000000000001125

54. Wei P, Zhang YG, Ling L, et al.: Effects of the short-term application of pantoprazole combined with aspirin and clopidogrel in the treatment of acute STEMI. Exp Ther Med. 2016, 12:2861-4. 10.3892/etm.2016.3693

55. Khan SU, Lone AN, Asad ZU, et al.: Meta-analysis of efficacy and safety of proton pump inhibitors with dual antiplatelet therapy for coronary artery disease. Cardiovasc Revasc Med. 2019, 20:1125-33. 10.1016/j.carrev.2019.02.002

56. Hu W, Tong J, Kuang X, Chen W, Liu Z: Influence of proton pump inhibitors on clinical outcomes in coronary heart disease patients receiving aspirin and clopidogrel: a meta-analysis. Medicine (Baltimore). 2018, 97:e9638. 10.1097/MD.0000000000009638

57. Jensen BES, Hansen JM, Larsen KS, Junker AB, Lassen JF, Jensen SE, Schaffalitzky de Muckadell OB: Randomized clinical trial: the impact of gastrointestinal risk factor screening and prophylactic proton pump inhibitor therapy in patients receiving dual antiplatelet therapy. Eur J Gastroenterol Hepatol. 2017, 29:111825. 10.1097/MEG.0000000000000934

58. Gargiulo G, Costa F, Ariotti S, et al.: Impact of proton pump inhibitors on clinical outcomes in patients treated with a 6- or 24-month dual-antiplatelet therapy duration: insights from the prolonging dualantiplatelet treatment after grading stent-induced intimal hyperplasia study trial. Am Heart J. 2016, 174:95102. 10.1016/j.ahj.2016.01.015

59. Zhu P, Gao Z, Tang XF, et al.: Impact of proton-pump inhibitors on the pharmacodynamic effect and clinical outcomes in patients receiving dual antiplatelet therapy after percutaneous coronary intervention: a propensity score analysis. Chin Med J (Engl). 2017, 130:2899-905. 10.4103/0366-6999.220304

60. Li Y, Ren X, Fang Z: Systematic review and meta-analysis: the effects of prophylactic proton pump inhibitor treatment in patients with coronary heart disease receiving dual antiplatelet therapy. J Cardiovasc Pharmacol. 2021, 77:835-61. 10.1097/FJC.0000000000001014

61. Shi WC, Gao SD, Yang JG, et al.: Impact of proton pump inhibitors on clinical outcomes in patients after acute myocardial infarction: a propensity score analysis from China Acute Myocardial Infarction (CAMI) registry. J Geriatr Cardiol. 2020, 17:659-65. 10.11909/j.issn.1671-5411.2020.11.008

62. Shi W, Yan L, Yang J, Yu M: Ethnic variance on long term clinical outcomes of concomitant use of proton pump inhibitors and clopidogrel in patients with stent implantation: a PRISMA-complaint systematic review with meta-analysis. Medicine (Baltimore). 2021, 100:e24366. 10.1097/MD.0000000000024366

63. Chandrasekhar J, Bansilal S, Baber U, et al.: Impact of proton pump inhibitors and dual antiplatelet therapy 


\section{Cureus}

cessation on outcomes following percutaneous coronary intervention: results from the PARIS registry. Catheter Cardiovasc Interv. 2017, 89:E217-25. 10.1002/ccd.26716

64. Tantry US, Bliden KP, Wei C, Storey RF, Armstrong M, Butler K, Gurbel PA: First analysis of the relation between CYP2C19 genotype and pharmacodynamics in patients treated with ticagrelor versus clopidogrel: the ONSET/OFFSET and RESPOND genotype studies. Circ Cardiovasc Genet. 2010, 3:556-66.

10.1161/CIRCGENETICS.110.958561

65. Kurlander JE, Barnes GD, Sukul D, et al.: Trials of dual antiplatelet therapy after percutaneous coronary intervention lack strategies to ensure appropriate gastroprotection. Am J Gastroenterol. 2021, 116:821-4. 10.14309/ajg.0000000000001134

66. Dinçer D, Ulukal Karancı E, Akın M, Adanır H: NSAID, antiaggregant, and/or anticoagulant-related upper gastrointestinal bleeding: is there any change in prophylaxis rate after a 10 -year period?. Turk J Gastroenterol. 2019, 30:505-10. 10.5152/tjg.2019.19057 